\title{
DEPENDENT AND INDEPENDENT CLUSTER COMPARISONS IN THE SUPERMATRIX
}

\author{
Orrin Cooper \\ University of Memphis \\ Memphis, TN, USA \\ E-mail: olcooper@memphis.edu \\ Guoqing Liu \\ University of Pittsburgh \\ Pittsburgh, PA, USA \\ E-mail: gul8@pitt.edu
}

\begin{abstract}
When designing an ANP model it is important for decision makers to acknowledge and properly address whether the elements in the models are dependent or independent of each other. If it is determined that the criteria and alternatives are dependent then criteria cluster weights should be obtained individually for each column in the Supermatrix. If criteria weights are applied broadly across large clusters or rows of the Supermatrix a compromising or restricting effect on the relative influences of the alternatives termed the "pigeonholing effect" can occur. Pigeonholing compromises the ratio preservation in the final priority vector and can lead to unintended results in the Limit matrix. A final priority vector with ratios that represent alternatives which are dependent on the criteria are best obtained by performing cluster comparisons individually for each column.
\end{abstract}

Keywords: ANP, Cluster Comparisons, Dependency 


\section{Introduction}

The Analytic Network Process (ANP), the general form of the Analytic Hierarchy Process (AHP), has been used to prioritize the alternatives within complex decisions in vast array of applications. This method, developed by Thomas Saaty, takes advantage of our natural ability to structure decisions as hierarchies or networks and make relative comparisons or judgments. When one decides to create an AHP or ANP model certain assumptions must be made about the how the decision at hand will be framed. Some of the questions one must consider are: What level of complexity should be captured? Is there inner and outer feedback in the model? Are there both tangible and intangible criteria? What is the meaning of the final priority vector that will be obtained? What level of dependence (or independence) is there among the criteria and alternatives?

The latter two questions about the meaning of the final priority vectors and level of dependence are worthy of addressing in further detail. Neither question has a universally correct answer but different answers produce different results. When a decision maker wishes to model a decision where the alternatives are entirely dependent on the defined

system or decision and obtain a priority vector of the form ${ }^{a_{i} / \sum_{i=1}^{n} a_{i}}$ in the Limit matrix, which provides the relative priority or contribution of an alternative $a_{i}$ with respect to the system of $n$ alternatives being considered careful attention must be paid to the weighting method used to convert an unweighted Supermatrix to a weighted Supermatrix. The pairwise comparisons to obtain the cluster weights must be performed for the criteria clusters in each column in the Supermatrix individually and not collectively. It has been shown that unique cluster weighting for each column, termed column normalization, must be used when tangible elements are considered and/or the model must be validated against actual results (Harker \& Vargas, 1990). However, as will be the application of column normalization is in fact independent of the tangibility of the elements considered.

If the criteria clusters are not weighted individually in each column a pigeonholing effect can occur where the alternatives' value or priorities are pigeonholed or restricted. The concept of pigeonholing and its effects will be discussed in greater detail. After the unintended consequences of pigeonholing are identified a solution is proposed, demonstrated, and the findings are generalized in a proof.

\section{Literature Review}

The ANP is the generalized form of the ANP; it allows for measuring dependence and feedback among alternatives and the criteria. When designing an ANP model a decision maker follows the same process as defined in Table 1 with two important differences. First, in step 2 the decision maker is not restricted to a hierarchal organization but may organize the criteria and alternatives into a network. A network design is a richer model that allows for the incorporation of inner and outer dependence among the criteria and alternatives (T. L. Saaty, 2005; T.L. Saaty \& Vargas, 2006). The second difference between the methods is that step 5 and 6 are separate processes in the ANP. Because the 
criteria are no longer assumed to be independent of the alternatives decision makers are have more flexibility in how they define the interactions and influence of the criteria and alternatives. In general this will lead to richer and more complex models under the ANP than the AHP. With these additional entries in the Supermatrix there may be multiple priority vectors in each column that sum to 1 . It is then necessary to perform comparisons among the criteria clusters to ensure that the weighted Supermatrix is column stochastic (T. L. Saaty, 2005). The weighted Supermatrix is then raised to powers to calculate the global priorities and weights. The ANP has been applied in a wide variety of contexts (Whitaker, 2007). Outside of the literature reviews the Encyclicons are also a valuable reference where readers can view a variety of different models under specific categories (T. L. Saaty \& Cillo, 2008; T. L. Saaty \& Ozdemir, 2005; T. L. Saaty \& Vargas, 2011).

While much has been written to dispute the validity of AHP/ANP (Belton \& Gear, 1983; J. S. Dyer, 1990; J. W. R. E. Dyer, 1985; Harker \& Vargas, 1990; T.L. Saaty, 1986; Thomas L. Saaty, 1990) the purpose of this publication is not to join these arguments for or against the AHP or ANP but rather to focus on problem definition at the outset of setting up a model. In particular to look at a subset of problems that if the user is looking for a priority vector of a specific form then a Fully-Dependent ANP model will obtain the desired vector. The form of this potentially desirable global priority vector $p=$ in the Limit matrix, which provides the relative priority or contribution of an alternative ai with respect to the system of $\mathrm{n}$ alternatives being considered. In other words, a FullyDependent model is one where the global priority vector depends on its contribution with respect the contributions of everything else in the system. In order to obtain a priority vector of the form , the criteria comparisons performed as part of step 6 Formulation of the weighted Supermatrix must be done individually for each alternative regardless of whether the criteria are tangible or intangible. In addition to the intangible properties of the criteria is worth noting two additional properties of ANP models. First, further investigation into the overall unit of measurement in an ANP model will allow criteria clusters to be combined into a general ANP model and facilitate comparisons across criteria clusters. (Choo, Schoner, \& Wedley, 1999; Wedley \& Choo, 2011) Second, the ability to compare criteria across clusters is further underscored by the findings of (Schoner et al., 1993) that all the elements in an ANP model are related. Third, in the current literature the tangibility of an object determines whether column normalization is required or not. Finally, an important property of the vectors that are combined in a Supermatrix further underscores the need for each column to be normalized individually. These properties and their application to a Fully-Dependent ANP model are explained in greater detail below.

Criteria weights in general are misunderstood and misused (Choo, Schoner, \& Wedley, 1999). Choo, et al. demonstrate that there is no consensus on the meaning or manner of deriving criteria weights. Furthermore they claim that criteria weights should not be calculated in a way that is independent of how they are used in a decision model. While criteria weights can be used for the normalization process, normalization in and of itself does not remove the units from the criteria being considered. According to Saaty (2004), relative scales do not need a unit of measurement. However, any multiplication by a constant $\mathrm{b} 0<\mathrm{b} \leq 1$, changes the unit of measure for a ratio vector. The fact that the value for a particular object has changed is evidence of a new unit being used. Wedley \& Choo (2011), explain that ratio scales in the ANP have a unit of measure and the unit of International Symposium of 3

Washington, D. C. the Analytic Hierarchy June 29 - July 2, 2014 
measure is important and useful. The unit of measurement is derived from the topmost node in the total network. The scale that one can obtain from such a unit is transient depending on the alternatives being considered but so is the ratio scale itself. Focusing on the ratios rather than the rank will improve the efficacy of the ANP. Wedley \& Choo (2011 p. 170), conclude "therein lie both the advantage and dilemma of AHP. We do not need explicit knowledge of the underlying unit of measure to derive a ratio scale, yet the derived scale has a unit." This understanding that the unit of measurement is derived from the topmost node in the network provides a unit to use as the basis for comparing criteria across clusters. Defining the unit of comparison facilitates comparing clusters together on individual level of each alternative with respect to their contribution to the overall network.

The design of the network emphasizes that the common unit of measurement is to be interpreted in the limit matrix. While in a network there is no topmost node of a network the following steps explain how a common unit is obtained (1) In the unweighted Supermatrix there are several units in each column - one unit sum scale for each priority vector of a set of comparisons. (2) In the weighted Supermatrix, each of unit sum vector of a column is linked to others so that they are commensurate within the column. (3) When the Supermatrix is then made stochastic, each column is in the unit of the totality of influences of each column. (4) In the limit matrix where all columns are the same, each column is in the unit of all influences of the entire Supermatrix.

\section{Hypotheses/Objectives}

A counter example and subsequent example are provided to motivate the method. A proof is provided to generalize the proposed method.

\section{Conclusions}

The AHP and ANP can be categorized as disruptive technology. They are amazing frameworks that are used to model independent criteria, semi-dependent criteria, and fully-dependent criteria as demonstrated above. Because column normalization requires additional pairwise comparisons it is crucial that decision makers determine upfront what is the final priority vector they wish to obtain supposed to represent. The need to address this question upfront is further underscored by the research discussed in the literature review regarding AHP and ANP. The column normalization put forth in this paper is not intended to be a universal approach to all ANP models; as was stated earlier, there are very good reasons to model the criteria independent or semi-dependent of the alternatives. The same can be said for modeling the criteria as fully-dependent on the alternatives.

What is important here is that if one wishes to model a fully-dependent system which will provide a priority vector of the form in the Limit matrix, which is interpreted as the relative priority or contribution of an alternative ai with respect to the system of $n$ alternatives being considered then column normalization must be performed. .

\section{Key References}


ISAHP Article: Cooper, Liu/ DEPENDENT AND INDEPENDENT CLUSTER COMPARISONS IN THE SUPERMATRIX To Be Submitted to the International Symposium of the Analytic Hierarchy Process 2014, Washington D.C., U.S.A.

Lipovetsky, S. (2011). AN INTERPRETATION OF THE AHP GLOBAL PRIORITY AS THE EIGENVECTOR SOLUTION OF AN ANP SUPERMATRIX. International Journal of the Analytic Hierarchy Process, 3(1).

Saaty, T. L. (1996). The Analytical Hierarchy Process (2nd ed. Vol. 1). Pittsburgh: RWS Publications.

Saaty, T.L., \& Vargas, L.G. (2006). Decision making with the analytic network process: Economic, political, social and technological applications with benefits, opportunities, costs and risks (Vol. 95): Springer.

Wedley, W.C. (2013). AHP/ANP BEFORE, PRESENT AND BEYOND. Paper presented at the ISAHP 201, Kuala Lumpar, Malaysia. Keynote Address

Wedley, W.C., \& Choo, E.U. (2001). A unit interpretation of multi-criteria ratios. Paper presented at the Proceedings of the sixth international symposium on the analytic hierarchy process, Berne, Switzerland.

Wedley, W.C., \& Choo, E.U. (2011). Multi-Criteria Ratios: What is the Unit? Journal of Multi-Criteria Decision Analysis, 18(3-4), 161-171. 